\title{
Interim observations on benefit/risk of azathioprine versus $D$-penicillamine in the treatment of rheumatoid arthritis
}

\author{
JOHN R WARD* AND H JAMS WILLIAMS* \\ From the School of Medicine, University of Utah, Salt Lake City, Utah, USA
}

Azathioprine has been shown to be an effective drug for the treatment of rheumatoid arthritis. Levy et al ${ }^{124}$ have reported improvement in joint counts with azathioprine and Urowitz et al ${ }^{224}$ found azathioprine to be superior to placebo in reducing the articular index and joint counts. Under double-blind conditions, azathioprine, cyclophosphamide and gold produced comparable clinical improvement over 18 months of observation. ${ }^{38}$ In a single-blind external observer trial, azathioprine $2.5 \mathrm{mg} / \mathrm{kg} /$ day produced similar efficacy and toxicity when compared with D-penicillamine $1 \mathrm{~g} /$ day. ${ }^{18}$ In a parallel uncontrolled open trial, azathioprine, gold and chloroquine were essentially equivalent in the control of disease activity in patients with early rheumatoid arthritis. ${ }^{56}$ Additional studies support the beneficial effects of azathioprine in rheumatoid arthritis. ${ }^{25} 1433225$

The selection of slow-acting antirheumatic drugs (SAARDs) for treatment still remains controversial. Our group considers gold to be the SAARD of first choice. However, for patients who fail to respond to gold, or develop toxicity, cytotoxic drugs or D-penicillamine represent the next therapeutic option. Thus, the current study was developed to compare the efficacy and toxicity of azathioprine and D-penicillamine in a prospective randomised controlled double-blind 24-week trial. The study will evaluate long-term efficacy and safety during a five-year non-blinded follow-up period, during which patients may continue to take one of the study drugs as long as it is clinically indicated.

\section{Materials and methods}

P A T I E N T S

A total of 200 patients will be entered. Patient selection criteria include the following.

(1) Definite or classical rheumatoid arthritis (ARA criteria) of greater than six months' duration.

*For the Cooperating Clinics, Cooperative Systematic Studies of the Rheumatic Diseases. Supported by NIAMDD Contract NO1-AM-6-2218 and a grant from Burroughs Wellcome Company.
(2) Onset after the age of 16 years.

(3) Active disease defined as having six or more swollen joints that are considered responsive to therapy and at least two of the following:

(a) Nine or more responsive joints tender on pressure;

(b) 45 minutes or more of morning stiffness;

(c) Westergren erythrocyte sedimentation rate of $28 \mathrm{~mm} / \mathrm{h}$ or greater.

(4) Failure to respond to at least $750 \mathrm{mg}$ parenteral gold or toxic reaction to gold.

(5) Inadequate control of arthritis with therapeutic doses of aspirin or other non-steroidal anti-inflammatory drugs (NSAIDs).

(6) Absence of childbearing potential in females.

(7) Absence of contraindication: hepatic disease, renal failure, proteinuria, thrombocytopenia or leucopenia

(8) No previous treatment with cytotoxic drugs.

(9) Constant optimal dose of aspirin, NSAID, and/or prednisone (10 $\mathrm{mg}$ or less/day).

(10) Informed consent.

T R E A T M E N T

A randomised schedule assigned 12 to 24 patients to each of 11 participating clinics, with equal numbers to receive azathioprine in doses of 1.25 to 1.5 $\mathrm{mg} / \mathrm{kg} /$ day or D-penicillamine in doses of 10 to 12 $\mathrm{mg} / \mathrm{kg} /$ day with a five-year follow-up. Each patient receives azathioprine tablets $(25 \mathrm{mg})$ and placebo capsules, or D-penicillamine capsules $(125 \mathrm{mg})$ and placebo tablets. The azathioprine is initiated to provide the full dose from start of trial, whereas D-penicillamine is increased by $250 \mathrm{mg}$ every four weeks until the full dose is reached and maintained for the 24 week duration of the double-blind phase.

MEASUREMENT OF DISEASE ACTIVITY For measurement of efficacy the following variables were measured at entry and every six weeks.

(1) Functional assessment.

(2) Duration of morning stiffness. 
(3) Onset of fatigue.

(4) Overall assessment of disease activity as estimated by both physician and patient.

(5) Joint counts for number of tender joints and tenderness score for joints $(0=$ none, $1=$ mild, $2=$ moderate, $3=$ severe).

(6) Joint counts for number of swollen joints and a swelling score.

Additional criteria for response include*:

(1) Remission.

(2) Significant clinical improvement.

(3) Overall meaningful clinical improvement.

(4) Subjective improvement.

(5) No response.

(6) Progressive disease.

Extra-articular manifestations, including subcutaneous nodules, skin ulcers, vasculitis, etc, will be evaluated.

\section{OTHER ASSESSMENTS}

Patients are seen every two weeks for evaluation of side-effects, monitoring of drug compliance and laboratory studies, which include complete blood count,

${ }^{*}$ Definitions will be provided in a report when the trial has been completed.

TABLE 1 Comparison of descriptive variable at entry for patients completing the trial by treatment group

\begin{tabular}{llcc}
\hline Variable & Measure & \multicolumn{2}{l}{ Treatment group } \\
\cline { 3 - 4 } & & $A(n=38)$ & $B(n=31)$ \\
\cline { 3 - 4 } & & 50 & 53 \\
Age (years) & Mean & 71 & 74 \\
Sex & Female (\%) & 84 & 90 \\
Race & Caucasian (\%) & 16 & 10 \\
& Black (\%) & 135 & 124 \\
Duration (months) & Mean & $2 \cdot 2$ & $2 \cdot 1$ \\
Functional class & Mean & $2 \cdot 2$ & $2 \cdot 1$ \\
Severity & Mean & & \\
\hline
\end{tabular}

TABLE 2 Median differences for measurement variables by treatment group between the conclusion and initiation of the trial* $^{*}$

\begin{tabular}{lcc}
\hline Variable & \multicolumn{2}{l}{ Treatment group } \\
\cline { 2 - 3 } & $A$ & $B$ \\
\hline Functional class (scale 1 = no disability, & & \\
$\quad$ 4=incapacitated) & 0.179 & 0.000 \\
Duration of morning stiffness (min) & 1.25 & -9.0 \\
Physician assessment (scale 1=no disease, & & \\
$\quad 4=$ severe) & 0.27 & 0.73 \\
Patient assessment (scale as above) & 0.68 & $0: 87$ \\
Number of tender joints & 6.50 & 10.33 \\
Joint tenderness score & 22.00 & 26.00 \\
Number of swollen joints & 5.00 & 10.00 \\
Joint swelling score & 5.52 & 7.00 \\
Grip strength (mmHg) & & \\
$\quad$ Right hand & 8.2 & 15.3 \\
Left hand & 5.3 & 13.0 \\
\hline
\end{tabular}

*Median change from entry to completion. A positive value indicates improvement. platelet count and urine analysis. A chemical survey and Westergren sedimentation rate is done every six weeks. Rheumatoid factor and antinuclear antibody tests are done at entry and completion.

\section{Results}

PATIENTS STUDIED

To date 148 of the 200 expected patients have been enrolled and 69 have completed the double-blind phase. Because the study is incomplete and still in progress, only blind comparisons of the two treatments are presented. The patients in each study group were comparable (table 1).

RESPONSE TO THERAPY

The results for selected measurements of disease activity are shown in table 2 . There was improvement in duration of morning stiffness, overall assessment of disease activity as judged by both physician and patient, number of tender joints and joint tenderness score and number of swollen joints and joint swelling score. The differences were similar but seemed to favour treatment B. No formal statistical testing has been done because of the ongoing nature of the trial

WIT H D R A W A L S

Of the 148 patients entered, 16 patients withdrew for the reasons shown in table 3 . More detailed reasons leading to withdrawal are shown in table 4 . Toxicity

TABLE 3 Reason for withdrawal of patients who have entered the trial to date

\begin{tabular}{lllll}
\hline Treatment & $\begin{array}{l}\text { No of patients } \\
\text { entered }\end{array}$ & \multicolumn{3}{l}{ No of patients withdrawn } \\
\cline { 3 - 5 } & ADR & NR & UD \\
\hline A & 77 & 6 & 0 & 2 \\
B & 71 & 5 & 1 & 2 \\
\hline ADR =adverse drug reaction & & & \\
NR=non-responsiveness \\
UD=unrelated to drug (moved, lack of co-operation, intercurrent illness, etc)
\end{tabular}

TABLE 4 Reason for withdrawal from study by treatment group

\begin{tabular}{lll}
\hline Reason for withdrawal & No of patients & \\
\cline { 2 - 3 } & Treatment $A$ & Treatment $B$ \\
\hline Thrombocytopenia & & 1 \\
Proteinuria & 2 & 1 \\
Leucopenia & 2 & 1 \\
Nausea & 1 & \\
Abnormal liver function tests & 1 & 1 \\
Itching and rash & & 1 \\
Oral ulcers & & 1 \\
Herpes zoster & & 1 \\
Inadequate response & 2 & 1 \\
Unrelated disease & & \\
Lack of co-operation & & \\
\hline
\end{tabular}


as determined by withdrawals was similar although from different causes for the two groups.

\section{Discussion}

Preliminary results of this study to compare the efficacy, safety and tolerance of selected doses of azathioprine and D-penicillamine have indicated improvement in rheumatoid arthritis with both treatments. However, the data to date would suggest better efficacy for treatment $B$. Until completion of the trial, blinding will be maintained. The null hypothesis states that one group is equal to the other group. Failure to reject the hypothesis when a real difference exists is termed a type II error, while rejection of the hypothesis when no real difference is present is a type I error. No formal statistical comparisons are presented because of the small sample size and thus the risk of committing a type II error. The trial was designed to detect a $30 \%$ difference between the treatment groups with $\alpha=0.05$ and $\beta=0 \cdot 1$, and allows for a dropout rate of $30 \%$. Until the 200 patients have completed the trial, only a striking difference in efficacy or toxicity will terminate the trial!

An important component of this study is to examine for a subset of unique responders. Thus, $a$ priori definitions of remission and varying degrees of clinical response and worsening were developed. Analysis of these subsets will also await completion of the formal trial.

The long-term follow-up of patients, who continue on their treatment for extended periods of time, is required to help the physician in the selection of treatment. Short-term efficacy is not always equatable to value of therapy, as tolerance and continued benefit are important.

It would have been far more useful to have been able to present not only the results of the double-blind comparison, but the long-term follow-up. While this is not possible, the ultimate results of this trial should help clinicians select which drug they judge would be helpful in the treatment of 'unresponsive' rheumatoid arthritis. 